\title{
Transient development of MHD natural convection flow in vertical concentric annulus
}

\author{
Basant K. Jha, Taiwo S. Yusuf \\ Department of Mathematics, Ahmadu Bello University, Nigeria
}

\begin{tabular}{l}
\hline \hline Article Info \\
\hline Article history: \\
Received Mar 29, 2019 \\
Revised Apr 20, 2019 \\
Accepted Mar 12, 2020 \\
\hline
\end{tabular}

\section{Keywords:}

Constant heating

Hartmann number Implicit finite difference

Natural convection

Riemann-sum approximation

\begin{abstract}
This paper examines the role of magnetic field on fully developed natural convection flow in an annulus due to symmetric of surfaces. The transport equations concerned with the model under consideration are rendered non-dimensional and transformed into the ordinary differential equation using Laplace transform technique. The solution obtained is then transformed to time domain using the Riemann-sum approximation approach. The governing equations are also solved using implicit finite difference method so as to establish the accuracy of the Riemann-sum approximation approach at transient as well as at steady state solution. The solutions obtained are graphically represented and the effects of pertinent parameters on the flow formation are investigated in detail. The Hartmann number $(M)$, is seen to have a retarding effect on the velocity, skin-frictions and the mass flow rate. Also, skin-friction at both surfaces and the mass flow rate within the annulus are found to be directly proportional to the radii ratio $(\lambda)$.
\end{abstract}

This is an open access article under the CC BY-SA license.

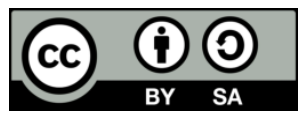

\section{Corresponding Author:}

Taiwo S. Yusuf,

Department of Mathematics,

Ahmadu Bello University,

Community Market, Zaira Nigeria, local 810211, Zaria, Nigeria.

Email: taiyeee@yahoo.com

\author{
NOMENCLATURE \\ $B_{0} \quad$ Constant magnetic flux density \\ $C_{p} \quad$ Specific heat at constant pressure \\ $g \quad$ Gravitational acceleration \\ $k \quad$ Thermal conductivity of the fluid \\ $M \quad$ Hartmann number \\ $\mathrm{Pr} \quad$ Prandtl number \\ $Q \quad$ Dimensionless mass flow rate \\ $r_{1} \quad$ Radius of the inner cylinder \\ $r_{2} \quad$ Radius of the outer cylinder \\ $r^{\prime} \quad$ Dimensional radial coordinate \\ $R \quad$ Dimensionless radial coordinate \\ $t \quad$ Dimensionless time \\ $t^{\prime} \quad$ Dimensional time \\ $T_{0} \quad$ Reference temperature \\ $T_{w} \quad$ Temperature at both surfaces \\ $u^{\prime} \quad$ Axial velocity \\ $U \quad$ Dimensionless axial velocity
}




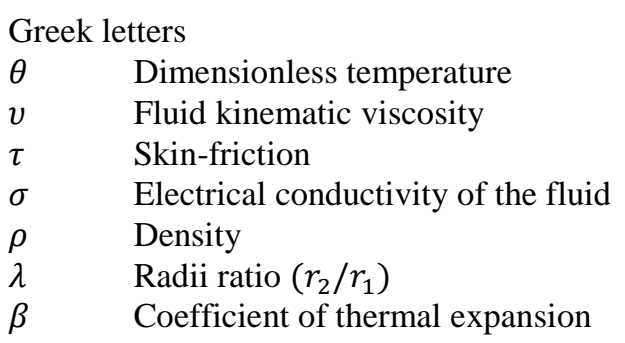

\section{INTRODUCTION}

In recent times, the natural convection with simultaneous heat and mass transfer flow of magnetohydrodynamics fluid has attracted many researchers in view of its various applications in astrophysics, geophysics, meteorology, aerodynamics, magnetohydrodynamics power generators and pumps, boundary layer control energy generators, accelerators, aerodynamics heating, polymer technology, petroleum industry, purification of crude oil, and in material processing such as metal forming, continuous casting wire, magnetohydrodynamics (MHD) bearings, MHD flow meters, geothermal energy excitations and plasma controls. Others include the study of stellar and solar structures, interstellar matter, and radio propagation through the ionosphere and so forth [1-9].

The study of MHD flow problem in an annulus was first discussed by Globe [10] who considered fully developed laminar MHD flow problem in an annulus. In his work, he studied the problem of steady flow of an electrical conducting incompressible fluid in an annular space between two infinitely long circular cylinders under the radial impressed magnetic fluid. Later on, Georgantopoulos and Goudas [11] studied the free convection effects on the hydromagnetic oscillatory flow in the Stokes problem past an infinite porous vertical limiting surface with constant suction. Again, the analytical solutions for transient fully developed natural convection in open-ended vertical concentric annulus was presented by Al-Nimr [12]. Later on, Al-Nimr [13] carried out analytical solutions for fully developed MHD natural-convection flow in the open-ended vertical concentric porous annulus. Al-Nimr and Database [14] presented the closed forms on transient fully developed free convection solutions, corresponding to four fundamental thermal boundary conditions in the vertical concentric annulus, while Sheikholeslami and Gorji-Bandpy [15] examined the numerical solution for free convection of ferrofluid in a cavity heated from below in the presence of external magnetic field. Sheikholeslami et al. [16] investigated MHD natural convection of nanofluid in a concentric annulus between a cold outer square cylinder and a heated inner circular.

In other work, Singh et al. [17] studied natural convection in vertical concentric annulus under a radial magnetic field, where they observed that both velocity and temperature are more in case of isothermal condition compared with constant heat flux case when gap between cylinders is less or equal to radius of inner cylinder. Nirmal et al. [18] studied an exact solution for unsteady magnetohydrodynamic free convection flow with constant heat flux and concluded that the magnetic field has a retarding effect on the velocity while the skin-friction at the plate increases with it. Furthermore, Jha et al. [19] considered fully developed MHD natural convection flow in a vertical microchannel with the effect of the transverse magnetic field in the presence of velocity slip and temperature jump at the annular micro-channel, they established that increase in curvature radius leads to an increase in the mass flow rate.

In a related article, Abbas et al. [20] investigated application of drug delivery in magnetohydrodynamics peristaltic blood flow of nanofluid in a non-uniform channel, while Bhatti et al. [21] considered combined effect of magnetohydrodynamics and partial slip on peristaltic blood flow of Ree-Eyring with wall properties. An analysis to investigate the combined effects of heat and mass transfer on free convection unsteady magnetohydrodynamics (MHD) flow of the viscous fluid embedded in a porous medium was later presented by Ali et al. [22]. Most recently, two-dimensional magnetohydrodynamic flow of a viscous fluid over a constant wedge immersed in a porous medium was studied by Kudenatti et al. [23], they found that MHD effects on the boundary layer are exactly the same as the porous medium in which both reduce the boundary layer thickness. This paper is devoted to investigate the role of magnetic field on fully developed natural convection flow of an incompressible and electrically conducting fluid filled between two vertical coaxial cylinders, when the two cylinders are subjected to constant heating.

\section{RESEARCH METHOD}

Consider a transient laminar fully developed natural convection flow of an incompressible, viscous and electrically conducting fluid in an annulus of infinite length under the influence of transverse magnetic field. The z-axis is taken along the axis of the cylinder in the vertically upward direction and $r^{\prime}$-axis is in 
the radial direction. A magnetic field of strength $B_{0}$ is assumed to be uniformly applied in the direction perpendicular to the direction of flow. In the present physical situation, the inlet fluid temperature is maintained at $T_{0}$, while a constant uniform heating of $T_{w}$ is applied at the outer surface of the inner cylinder and at the inner surface of the outer cylinder such that $T_{w}>T_{0}$ as presented in Figure 1. The flow is assumed to be fully developed both thermally and hydrodynamically, and the viscous dissipation, radiation, and compressibility effects are neglected. Following the work of Jha et al. [24] the momentum and energy equations governing the present physical situation is given by (1) and (2).

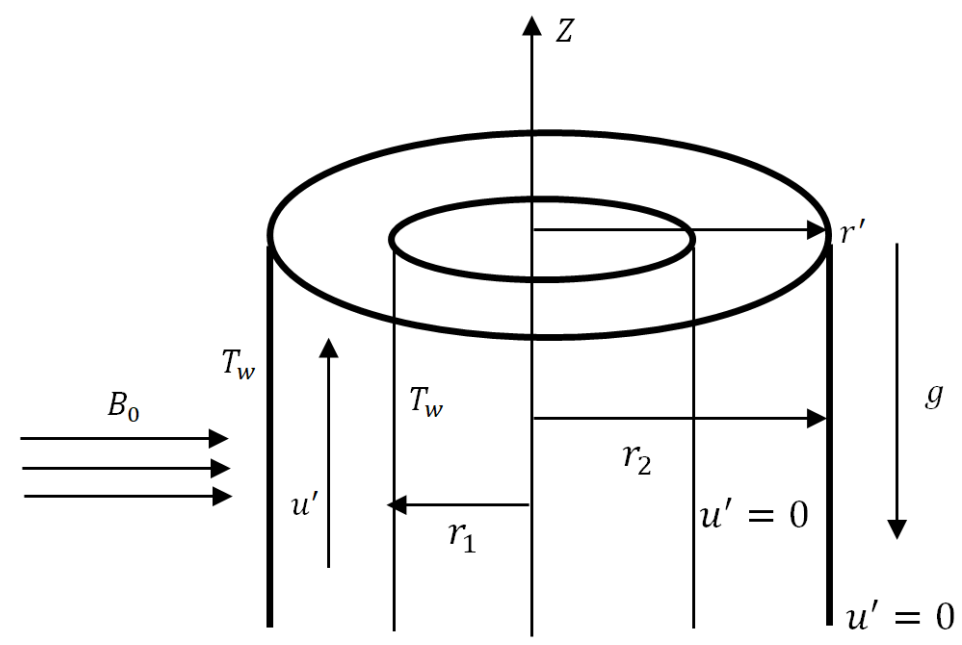

Figure 1. Schematic diagram of the problem

$\frac{\partial u^{\prime}}{\partial t^{\prime}}=v\left[\frac{\partial^{2} u^{\prime}}{\partial r^{\prime 2}}+\frac{1}{r^{\prime}} \frac{\partial u^{\prime}}{\partial r^{\prime}}\right]-\frac{\sigma B_{0}{ }^{2}}{\rho} u^{\prime}+g \beta\left(T^{\prime}-T_{0}\right)$

$\frac{\partial T^{\prime}}{\partial t^{\prime}}=\frac{k}{\rho c_{p}}\left[\frac{\partial^{2} T^{\prime}}{\partial r^{\prime 2}}+\frac{1}{r^{\prime}} \frac{\partial T^{\prime}}{\partial r^{\prime}}\right]$

The relevant dimensional initial and boundary conditions are;

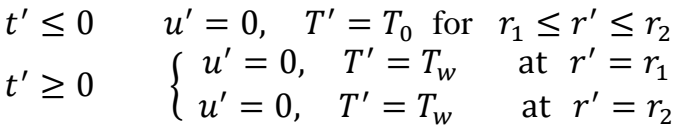

Introducing the following dimensionless quantities in (1) and (2).

$t=\frac{t^{\prime} v}{r_{1}{ }^{2}}, R=\frac{r^{\prime}}{r_{1}}, \lambda=\frac{r_{2}}{r_{1}}, M^{2}=\frac{\sigma B_{0}^{2} r_{1}^{2}}{\rho v}, \theta=\frac{\left(T^{\prime}-T_{0}\right)}{\left(T_{w}-T_{0}\right)}, \operatorname{Pr}=\frac{\mu c_{p}}{k}$

$U=u^{\prime} v\left[g \beta\left(T_{w}-T_{0}\right) r_{1}^{2}\right]^{-1}$

Equations (1) and (2) in dimensionless form are obtained as follows:

$\frac{\partial U}{\partial t}=\frac{\partial^{2} U}{\partial R^{2}}+\frac{1}{R} \frac{\partial U}{\partial R}-M^{2} U+\theta$

$\operatorname{Pr} \frac{\partial \theta}{\partial t}=\frac{\partial^{2} \theta}{\partial R^{2}}+\frac{1}{R} \frac{\partial \theta}{\partial R}$

The initial and boundary conditions in dimensionless form are:

$t \leq 0 \quad U=0, \quad \theta=0$ for $1 \leq R \leq \lambda$ 
$t>0 \quad\left\{\begin{array}{cc}U=0, \quad \theta=1 & \text { at } R=1 \\ U=0, \quad \theta=1 & \text { at } R=\lambda\end{array}\right.$

The physical quantities used in (1) to (6) are defined in the nomenclature. The solution of (5) and (6) with the associated initial and boundary conditions (7) and (8) can be obtained by using the Laplace transform technique. Defining the following transform variables.

$\bar{U}(R, s)=\int_{0}^{\infty} U(R, t) e^{-s t} d t, \quad \bar{\theta}(R, s)=\int_{0}^{\infty} \theta(R, t) e^{-s t} d t$,

Where the Laplace parameter, $(s>0)$ in (5) and (6) are transformed into the Laplace domain using the initial condition (7) to obtain.

$\frac{d^{2} \bar{U}}{d R^{2}}+\frac{1}{R} \frac{d \bar{U}}{d R}-\left(M^{2}+s\right) \bar{U}=-\bar{\theta}$

$\frac{d^{2} \bar{\theta}}{d R^{2}}+\frac{1}{R} \frac{d \bar{\theta}}{d R}-s \operatorname{Pr} \bar{\theta}=0$

Applying Laplace transform technique (9) on the boundary conditions (8), we have:

$\bar{U}=0, \quad \bar{\theta}=1 / s \quad$ at $R=1$

$\bar{U}=0, \quad \bar{\theta}=1 / s \quad$ at $R=\lambda$

The solution of (10) and (11) in Laplace domain subject to the boundary conditions (12) are:

$\bar{U}(R, s)=C_{3} I_{0}(R \delta)+C_{4} K_{0}(R \delta)-\left[\frac{C_{1} I_{0}(R \sqrt{s P r})+C_{2} K_{0}(R \sqrt{s P r})}{s(P r-1)-M^{2}}\right]$

$\bar{\theta}(R, s)=C_{1} I_{0}(R \sqrt{s P r})+C_{2} K_{0}(R \sqrt{s P r})$

Equation (13) and (14) are to be inverted in order to obtain their solutions in the time domain. Due to the complex nature of these inversions, we adopt a numerical procedure used in Jha and Yusuf [25] as well as Jha and Apere [26] which is based on the Riemann-sum approximation. According to this technique, any function in the Laplace domain can be inverted to the time domain as follows:

$P(R, t)=\frac{e^{\varepsilon t}}{t}\left[\frac{1}{2} \bar{P}(R, \varepsilon)+\operatorname{Re} \sum_{j=1}^{N} \bar{P}\left(R, \varepsilon+\frac{i j \pi}{t}\right)(-1)^{j}\right], 1 \leq R \leq \lambda$

where Re refers to the real part of $i=\sqrt{-1}$ the imaginary number. $\mathrm{N}$ is the number of terms used in the Riemann-sum approximation and $\varepsilon$ is the real part of the Bromwich contour that is used in inverting Laplace transforms. The Riemann-sum approximation for the Laplace inversion involves a single summation for the numerical process its accuracy depends on the value of $\varepsilon$ and the truncation error dictated by M. According to Tzou [27], the value of $\varepsilon t$ that best satisfied the result is 4.7 .

\subsection{Skin friction and mass flow rate}

The skin friction at $R=1, \bar{\tau}_{1}(R, s)$, and $R=\lambda, \bar{\tau}_{\lambda}(R, s)$ in Laplace domain is obtained by differentiating equations (13) and (14), respectively. While, the mass flow rate of the fluid through the annular gap in Laplace domain $\bar{Q}(R, s)$, is obtained by evaluating the integral $2 \pi \int_{1}^{\lambda} R \bar{U}(R, s) d R$. The solutions are as follows:

$$
\begin{aligned}
& \bar{\tau}_{1}=\left.\frac{d \bar{U}}{d R}\right|_{R=1}=\delta\left(C_{3} I_{1}(\delta)-C_{4} K_{1}(\delta)\right)-\sqrt{s P r}\left[\frac{C_{1} I_{1}(\sqrt{s P r})-C_{2} K_{1}(\sqrt{s P r})}{s(P r-1)-M^{2}}\right] \\
& \bar{\tau}_{\lambda}=-\left.\frac{d \bar{U}}{d R}\right|_{R=\lambda}=\delta\left(C_{4} K_{1}(\lambda \delta)-C_{3} I_{1}(\lambda \delta)\right)+\sqrt{s P r}\left[\frac{C_{1} I_{1}(\lambda \sqrt{s P r})-C_{2} K_{1}(\lambda \sqrt{s P r})}{s(P r-1)-M^{2}}\right] \\
& \bar{Q}=2 \pi \int_{1}^{\lambda} R \bar{U}(R, s) d R=2 \pi\left\{\left[\frac{C_{3}}{\delta}\left(\lambda I_{1}(\lambda \delta)-I_{1}(\delta)\right)-\frac{C_{4}}{\delta}\left(\lambda K_{1}(\lambda \delta)-K_{1}(\delta)\right)\right]-\right. \\
& \left.\frac{1}{s(P r-1)-M^{2}}\left[\frac{C_{1}}{\sqrt{s P r}}\left(\lambda I_{1}(\lambda \sqrt{s P r})-I_{1}(\sqrt{s P r})\right)-\frac{C_{2}}{\sqrt{s P r}}\left(\lambda K_{1}(\lambda \sqrt{s P r})-K_{1}(\sqrt{s P r})\right)\right]\right\}
\end{aligned}
$$


where $I_{0}, K_{0}, I_{1}, K_{1}$ are the modified Bessel function of first and second kind of order 0 and 1 respectively. In the same manner, the solutions are inverted to the time domain by applying the Riemann-sum approximation stated in (15).

\subsection{Validation of the method}

The accuracy of the Riemann-sum approximation approach in (14) is validated by computing the steady-state solution for the velocity field. This is obtained by taking $\frac{\partial()}{\partial t}=0$ in (5) and (6) which then reduces to the following ordinary differential equations. The implicit finite difference method has also been used to validate the Riemann-sum approximation approach, the advantage of this numerical procedure over others is that comparison can be made at both steady and transient state solution of the transport equations.

$\frac{d^{2} U}{d R^{2}}+\frac{1}{R} \frac{d U}{d R}-M^{2} U=-\theta$

$\frac{d^{2} \theta}{d R^{2}}+\frac{1}{R} \frac{d \theta}{d R}=0$

These are solved under the boundary conditions (8) to obtain the expressions for the steady-state velocity field, steady-state temperature field, steady-state skin frictions as well as the mass flow rate of the fluid. The solutions are respectively;

$$
\begin{aligned}
& U(R)=C_{5} I_{0}(M R)+C_{6} K_{0}(M R)+\frac{1}{M^{2}} \\
& \theta_{S}(R)=1 \\
& \tau_{1 s}=\left.\frac{d U}{d R}\right|_{R=1}=M\left(C_{5} I_{1}(M)-C_{6} K_{1}(M)\right) \\
& \tau_{\lambda s}=-\left.\frac{d U}{d R}\right|_{R=\lambda}=M\left(C_{6} K_{1}(\lambda M)-C_{5} I_{1}(\lambda M)\right) \\
& Q_{s}=2 \pi \int_{1}^{\lambda} R U(R, t) d R=2 \pi\left[\frac{C_{5}}{M}\left(\lambda I_{1}(\lambda M)-I_{1}(M)\right)-\frac{C_{6}}{M}\left(\lambda K_{1}(\lambda M)-K_{1}(M)\right)+\frac{1}{2 M^{2}}\left(\lambda^{2}-1\right)\right]
\end{aligned}
$$

The constants $C_{5}$ and $C_{6}$ in (25) are stated by:

$$
\begin{aligned}
& \delta=\sqrt{M^{2}+s} \\
& E 1=s\left[I_{0}(\lambda \sqrt{s P r}) K_{0}(\sqrt{s P r})-I_{0}(\sqrt{s P r}) K_{0}(\lambda \sqrt{s P r})\right] \\
& E 2=s\left[s(P r-1)-M^{2}\right]\left[I_{0}(\delta) K_{0}(\lambda \delta)-I_{0}(\lambda \delta) K_{0}(\delta)\right] \\
& E 3=M^{2}\left[I_{0}(\lambda M) K_{0}(M)-I_{0}(M) K_{0}(\lambda M)\right] \\
& C_{1}=\frac{\left[K_{0}(\sqrt{s P r})-K_{0}(\lambda \sqrt{s P r})\right]}{E 1} \\
& C_{2}=\frac{\left[I_{0}(\lambda \sqrt{s P r})-I_{0}(\sqrt{s P r})\right]}{E 1} \\
& C_{3}=\frac{\left[K_{0}(\lambda \delta)-K_{0}(\delta)\right]}{E 2} \\
& C_{4}=\frac{\left[I_{0}(\lambda \delta)-I_{0}(\delta)\right]}{E 2} \\
& C_{5}=\frac{\left[K_{0}(\lambda M)-K_{0}(M)\right]}{E 3} \\
& C_{6}=\frac{\left[I_{0}(M)-I_{0}(\lambda M)\right]}{E 3}
\end{aligned}
$$

The numerical values of the velocity obtained using the Riemann-sum approximation approach, implicit finite difference method and those obtained from the exact solution of the steady-state choosing value of $M=2, \lambda=2$. Is presented in Table 1 . The comparison between the results, shows that at large time (steady-state) there is an excellent agreement between the Riemann-sum approximation approach and the implicit finite difference. 
Table 1. Numerical values of the velocity obtained using Riemann-sum approximation approach, implicit finite difference and exact solution for different values of $\mathrm{R}$

\begin{tabular}{ccccc}
\hline \multirow{2}{*}{$\mathrm{t}$} & $\mathrm{R}$ & $\begin{array}{c}\text { Riemann-sum } \\
\text { approximation }\end{array}$ & $\begin{array}{c}\text { Implicit finite } \\
\text { Difference }\end{array}$ & $\begin{array}{c}\text { Exact } \\
\text { solution }\end{array}$ \\
\hline \multirow{nnyyy}{0.2}{} & 1.2 & 0.0478 & 0.0478 & 0.0624 \\
& 1.4 & 0.0652 & 0.0651 & 0.0870 \\
& 1.6 & 0.0633 & 0.0632 & 0.0837 \\
0.4 & 1.8 & 0.0434 & 0.0434 & 0.0553 \\
& 1.2 & 0.0608 & 0.0608 & 0.0624 \\
& 1.4 & 0.0846 & 0.0846 & 0.0870 \\
& 1.6 & 0.0814 & 0.0814 & 0.0837 \\
& 1.8 & 0.0540 & 0.0540 & 0.0553 \\
& 1.2 & 0.0623 & 0.0623 & 0.0624 \\
& 1.4 & 0.0868 & 0.0868 & 0.0870 \\
& 1.6 & 0.0835 & 0.0835 & 0.0837 \\
& 1.8 & 0.0552 & 0.0552 & 0.0553 \\
Steady state & 1.2 & 0.0624 & 0.0624 & 0.0624 \\
& 1.4 & 0.0870 & 0.0870 & 0.0870 \\
& 1.6 & 0.0837 & 0.0837 & 0.0837 \\
& 1.8 & 0.0553 & 0.0553 & 0.0553 \\
\hline
\end{tabular}

\section{RESULTS AND DISCUSSION}

In order to have a clear insight of the physical problem under consideration, a numerical computation is performed using the mathematical laboratory software (MATLAB) to compute and generate graphs for the velocity field, temperature field, skin-frictions and mass flux for different values of the governing parameters, so as to comment on their relative contribution to the flow formation. In this work, two different cases of fluid are been examined these include air with $\operatorname{Pr}=0.71$ and water with $\operatorname{Pr}=7.0$. The effect of variation of the governing parameters $M, \operatorname{Pr}$ and $\lambda$ on the flow formations are presented in Figures 2-9. Unless otherwise stated, the value $t=0.2, M=2$ and $\lambda=2$ are selected arbitrarily to study the effect of various parameters on the flow behavior. The influence of Prandtl number and time on the temperature profiles is shown in Figure 2. It is revealed in Figure 2 that fluid temperature increases as $P r$ and $t$ increases. It is concluded from Figure 2 that the reduction in fluid temperature is directly proportional to the decrease in thermal diffusivity. Figure 3 shows that an increase in the values of the Hartmann number $M$ causes retardation to the fluid flow indicating the fact that the imposition of magnetic field slow down the flow.

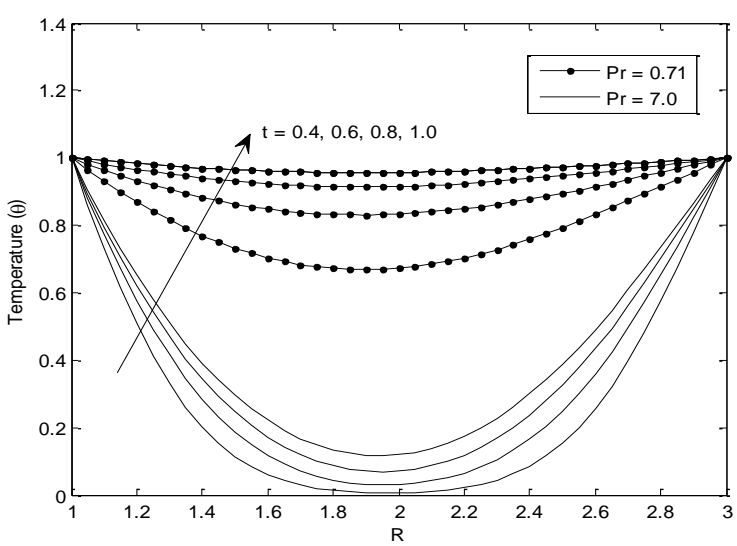

Figure 2. Temperature distribution for different values of $t(M=2.0)$

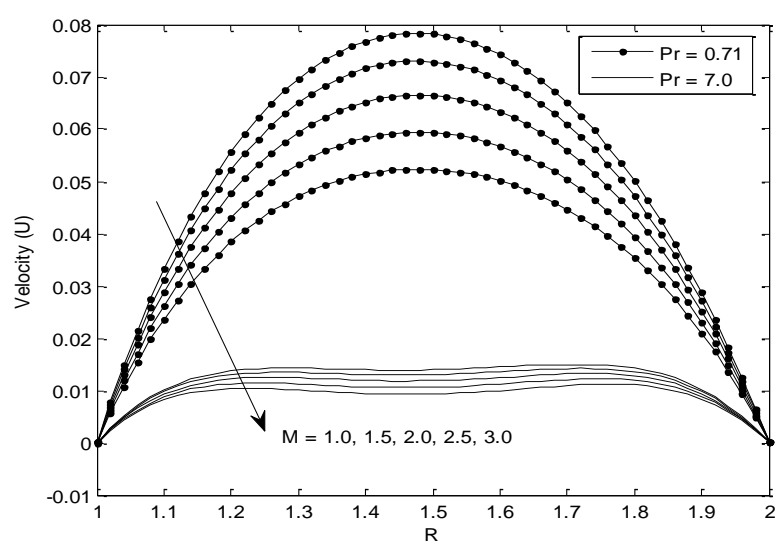

Figure 3. Velocity distribution for different values of $M(\mathrm{t}=0.2)$

This remark is consistent with the physical fact that the Lorentz force that appears due to the interaction of the magnetic field and the fluid velocity resists the corresponding fluid flow, resulting in the velocity to decrease gradually. Figure 3 also indicates how the velocity field is affected corresponding to an increase in the values of $P r$. We recall that an increase in $\operatorname{Pr}$ in Figure 2 signifies a fall in thermal diffusivity for the model under consideration. It is learnt from Figure 3 that when the thermal diffusivity of the fluid is reduced, the flow gets decelerated largely which may be attributed to the fact that a low thermal diffusivity leads to a corresponding decrease in the kinetic energy of the molecules of the fluid, which in turn 
affects the fluid velocity adversely. Figure 3 also revealed that the fluid velocity increases with increase in time till it attains steady state. It is worthy to note that $\operatorname{Pr}$ has no effect on the velocity and temperature profiles at steady state.

Figures 4 and 6 present variation of skin-friction at outer surface of the inner cylinder $(R=1)$ and the inner surface of the outer cylinder $(R=\lambda)$ respectively for different values of the Hartmann number $(M)$. It is obvious from these Figures 4 and 6 that skin-friction decreases with increase in Hartmann number $(M)$ on both surfaces for both cases of $\operatorname{Pr}$. Fluid with $\operatorname{Pr}=0.71$ is observed to induces higher friction at both surfaces of the cylinders in comparison with fluid with $\operatorname{Pr}=7.0$ which is physically true, since higher velocity results in friction at the walls. In addition, it is clear that skin friction at both cylinders for air attains steady state faster than water. This suggests that if one considers to reduce the friction at the surfaces, fluids with higher Prandtl numbers like water $(\mathrm{Pr}=7)$ should be considered. Variation of skinfriction profiles at the outer surface of the inner cylinder and the inner surface of the outer cylinder for different values of radii ratio $(\lambda)$ are shown respectively in Figures 5 and 7. It is obvious from both Figures 5 and 7 that skin-friction on both cylinder increases with $(\lambda)$. A keen scrutiny of the Figures 5 and 7 reveal that the skin-friction is independent of time for $\mathrm{Pr}=0.71$ except as $t$ tends to zero.

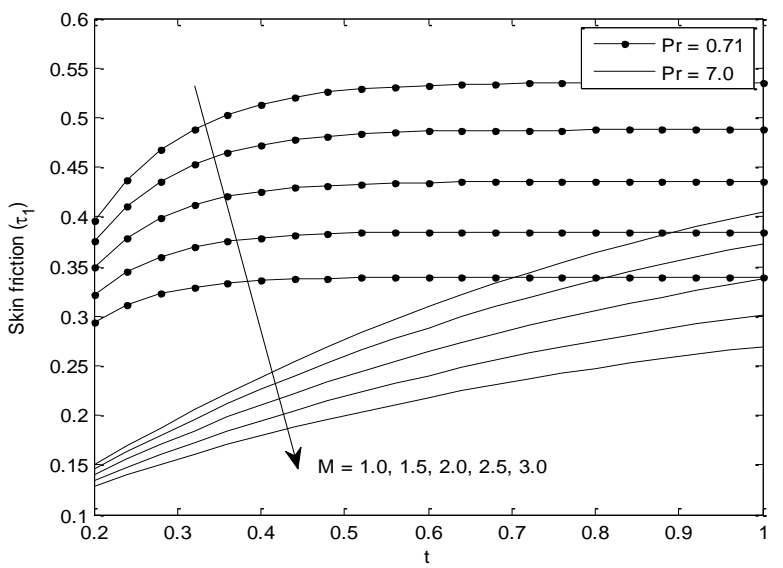

Figure 4. Variation of skin friction $\left(\tau_{1}\right)$ for different values of $M$

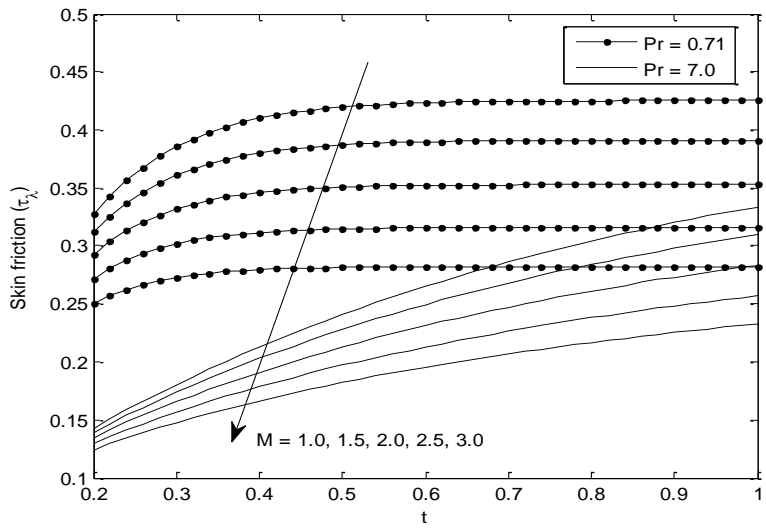

Figure 6. Variation of skin friction $\left(\tau_{\lambda}\right)$ for different values of $\mathrm{M}(\lambda=2)$

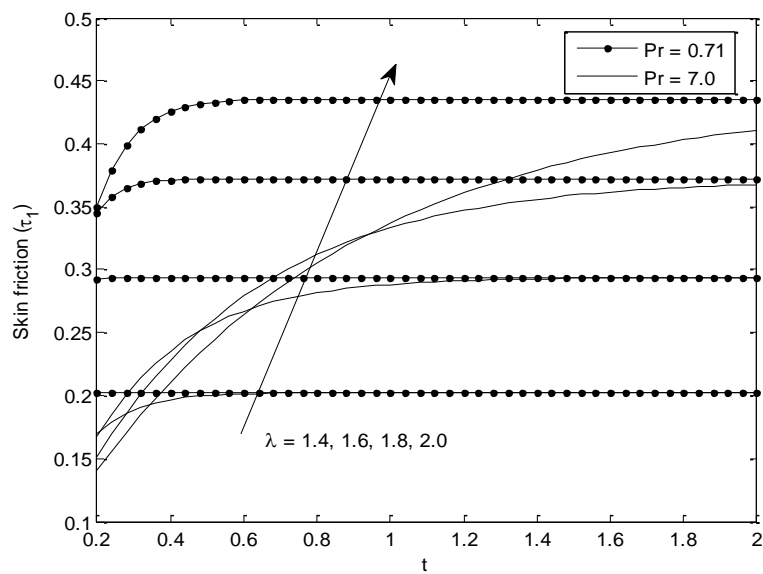

Figure 5. Variation of skin friction $\left(\tau_{1}\right)$ for different values of $\lambda(M=2)$

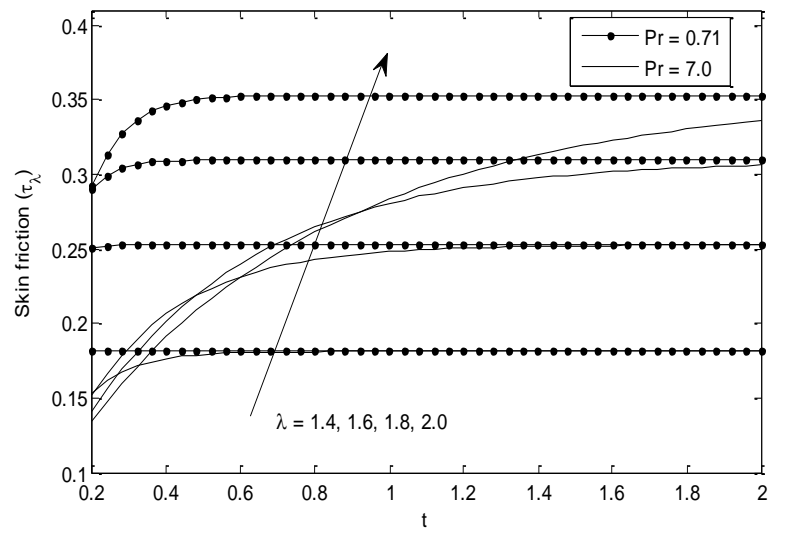

Figure 7. Variation of skin friction $\left(\tau_{\lambda}\right)$ for different values of $\lambda(M=2)$

Figure 8 reveals the mass flow rate plotted against Hartmann number $(M)$ for different values of time $(t)$. It is observed that the mass flow rate decreases as Hartmann number $(M)$ increases but increases with time $(t)$. Figure 9 illustrations variation of mass flow rate for different values of radii ratio $(\lambda)$. It is evident that mass flow rate increases with increase in radii ratio $(\lambda)$ for both cases of $(\operatorname{Pr})$. It is worthy to 
note that mass flow rate is constant for different values of radii ratio $(\lambda)$ in the case $\operatorname{Pr}=0.71$, but increases with time $(t)$ for $\operatorname{Pr}=7.0$. Figures 8 and 9 lead us to conclude that the parameters $\operatorname{Pr}$ and $M$ have significant contributions in regulating the amount of total discharge of fluid through the annulus and they may be suitably chosen to control the mass flux.

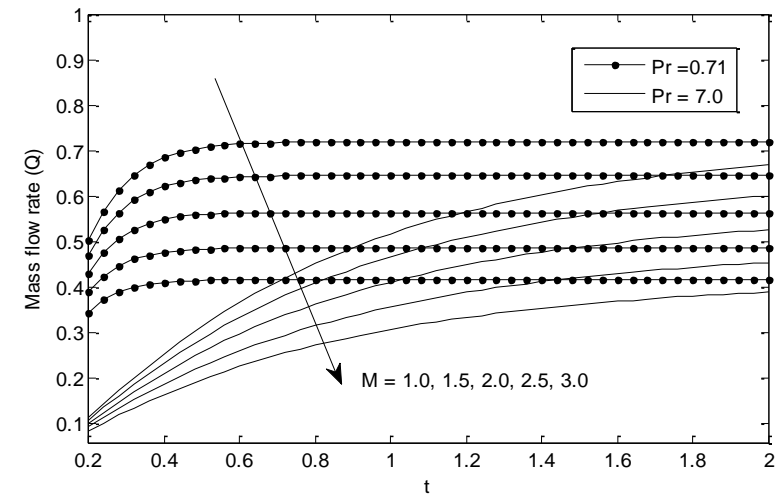

Figure 8. Variation of mass flow rate $(Q)$ for different values of $M(\lambda=2)$

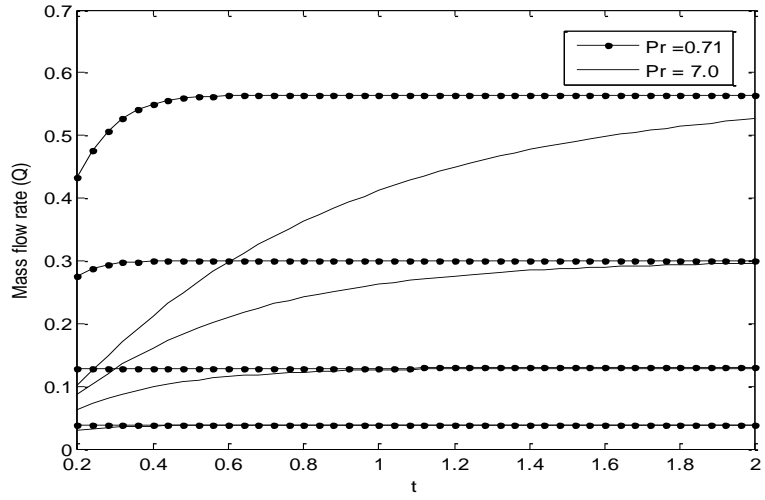

Figure 9. Variation of mass flow rate $(Q)$ for different values of $\lambda(M=2)$

\section{CONCLUSION}

A semi-analytical study is conducted to examine the role of magnetic field on an incompressible and an electrically conducting fluid filled within two coaxial cylinders. The Laplace transform technique and Riemann-sum approximation method have been used to obtain the solution of the governing equations. The influence of Hartmann number $(M)$, Prandtl $(P r)$, radii ratio $(\lambda)$, and time $(t)$ on the velocity field, temperature distribution, skin frictions and mass flow rate have been extensively discussed. The main findings in the present research are:

a. It is found that an increase in Hartmann number $(M)$ has a retarding effect on the velocity field, mass flow rate and skin-friction at both surfaces.

b. It is worthy to conclude that an increase in the radii ratio $(\lambda)$ increases the skin-friction at both surfaces.

c. Air is established to have higher fluid velocity, mass flow rate and skin-friction on both surfaces in comparison with water.

d. Generally, Air $(\operatorname{Pr}=0.71)$ attains steady state temperature faster, due to its higher thermal diffusivity (See Figure 3) in comparison with water $(\operatorname{Pr}=7.0)$.

e. Skin-friction at both surfaces and Mass flow rate are seen to be independent of time except at small value of time $(t)$.

\section{REFERENCES}

[1] P. Chandran, N. Sacheti, and A. K. Singh, "A unified approach to analytical solution of a hydromagnetic free convection flow," Scientiae Mathematicae Japonicae, vol. 53, no. 3, pp. 467-476, 2001.

[2] D. T. Swift-Hook and J. K. Wright, "The constant-Mach-number MHD generator," Journal of Fluid Mechanics, vol. 15 , no. 1 , pp. $97-110,1963$.

[3] A. A. Kaufman and G. V. Keller, "The Magnetotelluric Sounding Method,” Elsevier, New York, NY, USA, 1981.

[4] Y. Aoki, T. Seidou, and N. Ohtomo, "Measurement of the time dependent temperature variation of combustion MHD plasma," Japanese Journal of Applied Physics, vol. 23, no. 12, pp. 1628-1633, 1984.

[5] J. E. Borovsky, R. C. Elphic, H. O. Funsten, and M. F. Thomsen, "The Earth's plasma sheet as a laboratory for flow turbulence in high- $\beta$ MHD MHD," Journal of Plasma Physics, vol. 57, no. 1, pp. 1-34, 1997.

[6] D. M. Bushnell and C. B. McGinley, "Turbulence control in wall flows, Annual Review of Fluid" Mechanics, vol. 21, pp. 1-20, 1989.

[7] T. Hayat and Z. Abbas, "Heat transfer analysis on the MHD flow of a second grade fluid in a channel with porous medium," Chaos, Solitons and Fractals, vol. 38, no. 2, pp. 556-567, 2008.

[8] M. M. Rahman and M.A. Sattar, "Magnetohydrodynamics convective flow of a micropolar fluid past a continuously moving vertical porous plate in the presence of heat generation/absorption," Journal of Heat Transfer, vol. 128, no. 2, pp. 142-152, 2006.

[9] M. Kaviany, "Boundary-layer treatment of forced convection heat transfer from a semi-infinite flat plate embedded in porous media," Journal of Heat Transfer, vol. 109, no. 2, pp. 345-349, 1987. 
[10] S. Globe, "Laminar steady-state Magnetohydrodynamic flow in an annular channel," Physics of Fluids, vol. 2, no. 4, pp. 404-407, 1959.

[11] N. G. Kafousias, C. V. Massalas, A. A. Raptis, G. J. Tzivanidis, G. A. Georgantopoulos, and G. L. Goudas, "Free convection effects on the hydromagnetic oscillatory flow in the Stokes problem past an infinite porous vertical limiting surface with constant suction," Astrophys. Space Sci., vol. 68, no. 1, pp. 99-110, 1980.

[12] M. A. Al-Nimr, "Analytical solution for transient laminar fully developed free convection in vertical concentric annulus," Int. J. Heat Mass Transfer, vol. 36, no. 9, pp. 2385-2395, 1993.

[13] M. A. Al-Nimr, "MHD free-convection flow in open-ended vertical concentric porous annulus," Appl. Energy, vol.50, no. 4, pp. 293-311, 1995.

[14] M. A. Al-Nimr and T. Darabseh, "Analytical solution for transient laminar fully developed free convection in open-ended vertical concentric porous annulus," J. Heat Transfer (ASME), vol. 117, no. 3, pp. 762-764, 1995.

[15] M. Sheikholeslami and M. Gorji-Bandpy, "Free convection of ferrofluid in a cavity heated from below in the presence of an external magnetic field," Powder Technol., vol. 256, pp. 490-498, 2014.

[16] M. Sheikholeslami, M. Gorji-Bandpy, and D. D. Ganji, "Lattice Boltzmann method for MHD natural convection heat transfer using nanofluid," Powder Technol., vol. 254, pp. 82-93, 2014.

[17] S. K. Singh et al., "Natural convection in vertical concentric annulus under a radial magnetic field," Heat and Mass Transfer, vol. 32, pp. 399-401, 1997.

[18] C. S. Nirmal and C. Palleth, "An exact solution for unsteady magnetohydrodynamic free convection flow with constant heat flux," Heat and Mass Transfer, vol. 21, no. 1, pp. 131-142, 1994.

[19] B. K. Jha, B. Aina and S. Isa, "Fully developed MHD natural convection flow in a vertical annular microchannel: An exact solution," Journal of King Saud University -Science, vol. 27, no. 3, pp. 253-259, 2015.

[20] M. A. Abbas, Y. Q. Bai, M. M. Rashidi, and M. M. Bhatti, "Application of drug delivery in magnetohydrodynamics peristaltic blood flow of nanofluid in a non-uniform channel," Journal of Mechanics in Medicine and Biology, vol. 16, no. 4, pp. 1650052, 2016.

[21] M. M. Bhatti, M. A. Abbas, and M. M. Rashidi, "Combined effect of magnetohydrodynamics and partial slip on peristaltic blood flow of Ree-Eyring with wall properties," Engineering Science and Technology, an International Journal., vol. 19, no. 3, pp. 1497-1502, 2016.

[22] F. Ali, I. Khan, S. Shafie, and N. Musthapa, "Heat and mass transfer with free convection MHD flow past a vertical plate embedded in a porous medium," Math. probl. in eng., vol. 2013, no. 3, pp. 1-13, 2013.

[23] R. B. Kudenatti, S. R. Kirsur, A. L. Nargund, and N. M. Bujurke, "Similarity Solutions of the MHD Boundary Layer Flow Past a Constant Wedge within Porous Media," Math. Probl. in Eng., vol. 2017, pp. 1-11, 2017.

[24] B. K. Jha, A. K. Singh and H. S. Takhar, "Transient free convective flow in a vertical channel due to symmetric heating," Int. J. Appl. Mech. Eng, vol. 8, no. 3, pp. 497-502, 2003.

[25] B. K. Jha and T.S. Yusuf, Transient free convective flow in an annular porous medium: A semi-analytical approach, Eng. Sci. and Tech., an Int. J., vol. 19, no. 4, pp. 1936-1948, 2016.

[26] B. K. Jha, and C.A. Apere, "Unsteady MHD Couette flow in an annulus, the Riemann-sum approximation approach," J Phys Soc Jpn., vol. 79, no. 12, pp. 1-5, 2007.

[27] D. Y. Tzou, "Macro to microscale heat transfer: the lagging behavior," London, Taylor and Francis, 1997.

\section{BIOGRAPHIES OF AUTHORS}

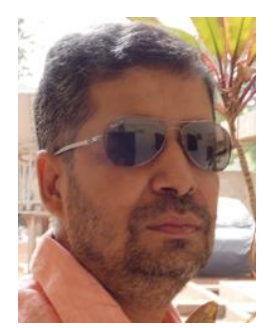

Basant Kumar Jha was born in Darbhanga, Bihar, India. He is a professor in applied mathematics at Ahmadu Bello University, Zaria, Nigeria. He has published more than 140 papers in reputed national/international journals. His research interest includes flow through porous media, magnetohydrodynamics, computational fluid dynamics, and heat and mass transfer.

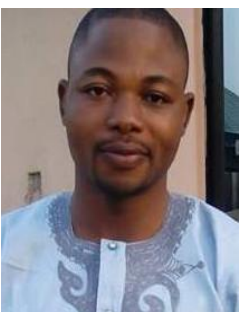

Yusuf Samuel Taiwo was born in Ikoyi, Kogi State, Nigeria. He is a lecturer and currently a PhD student in the Department of Mathematics Ahmadu Bello University (ABU), Zaria, Nigeria. He had his B.Sc. (first class honours) in the Department of Mathematics, Abu Zaria (2010). His areas of interest include flow through porous media, computational fluid dynamics, as well as heat and mass transfer. 